\title{
Influence of Rehabilitation on Oxygen Uptake Kinetics during High Intensity Exercise in Patients with Idiopathic Pulmonary Fibrosis
}

\author{
Sameera Peumal Senanayake, ${ }^{1,2}$ Kim Harrison ${ }^{3}$, Simon Hilldrup ${ }^{3}$, Michael Lewis ${ }^{1}$ \\ ${ }^{1}$ Swansea University, Swansea, UK \\ ${ }^{2}$ General Sir John Kotelawala Defence University, Ratmalana, Sri Lanka \\ ${ }^{3}$ Respiratory Unit, Morriston Hospital, Abertawe Bro Morgannwg University Health Board, Swansea, UK \\ Email: sp.senanayake@kdu.ac.lk,m.j.lewis@swansea.ac.uk
}

How to cite this paper: Senanayake, S.P., Harrison, K., Hilldrup, S. and Lewis, M. (2020) Influence of Rehabilitation on Oxygen Uptake Kinetics during High Intensity Exercise in Patients with Idiopathic Pulmonary Fibrosis. Open Journal of Respiratory Diseases, 10, 59-74.

https://doi.org/10.4236/ojrd.2020.104007

Received: August 14, 2020

Accepted: September 19, 2020

Published: September 22, 2020

Copyright $\odot 2020$ by author(s) and Scientific Research Publishing Inc. This work is licensed under the Creative Commons Attribution International License (CC BY 4.0).

http://creativecommons.org/licenses/by/4.0/

\begin{abstract}
Idiopathic Pulmonary Fibrosis (IPF) is a chronic, progressive and life-limiting condition of unknown cause with no curative treatment. The impact of IPF on a patient's quality of life is devastating and palliative treatment such as pulmonary rehabilitation programmes are used to improve quality of life among these individuals, yet relatively little attention has been made to access the effectiveness of rehabilitation programs aimed designed for these patients. There are large gaps in our knowledge on the cardiorespiratory response to exercise and rehabilitation among IPF patients and this study aims to fill this gap in a physiological prospective. We quantified the effects of an eight-week pulmonary rehabilitation program for IPF patients, conducted at Morriston Hospital, Swansea. Fifteen individuals (13 with Idiopathic Pulmonary Fibrosis and two with Pulmonary Fibrosis associated with Rheumatoid Disease) underwent physical and physiological assessments during a three-day testing protocol: 1) On Day 1, physical function (six-minute walk test) and ventilatory function (spirometry) were measured; 2) On Day 2, patients' cardio-respiratory responses to slowly-increasing, wide-ranging metabolic challenge (using a protocol consisting of periods of rest, incremental bicycle exercise to maximal effort, and post-exercise recovery) were assessed via respiratory gas analysis and ECG recording; 3) On Day 3, patients' cardio-respiratory responses to rapid-onset, high-workload metabolic challenge were assessed (using a protocol including a rapid-onset, constant workload bicycle exercise) by modelling dynamic respiratory oxygen and heart rate responses. Respiratory gas analysis was used to measure the rate of oxygen uptake $\left(\dot{\mathrm{V}}_{2}\right)$ and oxygen uptake efficiency (OUES). All assessments were performed before and after participants completed the pulmonary rehabilitation programme. A Holter
\end{abstract}


ECG recorder (Pathfinder/Lifecard Digital CF system; Spacelabs Medical Ltd., UK) provided continuous ECG data throughout each exercise test, from which heart rate was derived. Following the rehabilitation programme, heart rate was elevated by $11 \%-18 \%$ during exercise and recovery states. Post-rehabilitation $\dot{\mathrm{V}}_{2}$ was significantly increased $(\mathrm{p}=0.01-0.03)$ during the first two minutes of heavy-intensity exercise, whilst HR was reduced $(\mathrm{p}=0.04)$ during this period. OUES and standard measures of respiratory performance (minute ventilation, peak $\mathrm{V}_{2}$ ) were unchanged following rehabilitation, whilst peak HR and work rates were significantly reduced during incremental exercise only $\left(\mathrm{p}<10^{-3}\right)$. Pulmonary rehabilitation improved the rate of oxygen uptake during heavy-intensity exercise, despite substantially lower heart rates. This suggests that the rehabilitation programme increased systemic arterial-tissue oxygen exchange and/or influenced cardiovascular function to improve systemic oxygen delivery. We might therefore expect that individuals with IPF would find it easier to perform the activities of daily life, including those requiring substantial metabolic demands, following rehabilitation.

\section{Keywords}

IPF, $\mathrm{VO}_{2}$, OUES, Rehabilitation, Physiology

\section{Introduction}

Idiopathic Pulmonary Fibrosis (IPF) is the commonest of the idiopathic interstitial pneumonias. Its cause is unknown, but it causes progressive fibrosis of the alveolar walls and severe impairment of lung function [1] (Raghu et al., 2011). The median survival for patients diagnosed with IPF is 2.5 to 3.5 years but the range is wide [2] (Ley, Collard, \& King, 2011), with some individuals following a slowly declining trajectory over many years and others experiencing a rapid disease progression. The histological characteristics of IPF include excess extracellular matrix within the alveolar interstitium and fibroblastic foci in a sub-pleural distribution are described as usual interstitial pneumonia (UIP), although similar pathological changes are sometimes observed in patients with collagen vascular disease such as rheumatoid arthritis. Patients with UIP present with symptoms of increasing breathlessness and fatigue which results in difficulty conducting day-to-day activities and a reduced quality of life [3] (Gross \& Hunninghake, 2001).

Whilst new anti-fibrotic therapies that slow the decline in lung function have recently been recommended for people with IPF [1] (Raghu et al., 2011) national guidelines recommend pulmonary rehabilitation (PR) to improve functional performance. However, whilst pulmonary rehabilitation is known to improve exercise performance and lung function in other forms of lung disease such as chronic obstructive pulmonary disease (COPD) [4] (Spruit, 2013), evidence of its benefit in IPF is limited [5] (Bolton, 2013) but growing. For example, two re- 
cent studies reported improved six-minute walk distance (6 MWD) and improvement in both health-related quality of life (HRQoL) and symptom severity following eight weeks PR [6] (Dowman, 2014) [7] (Tonelli et al., 2017). In both studies, patients with the most severe functional impairment at baseline appeared to benefit most although the reasons for this are unclear. Further research is therefore needed to investigate the possible physiological mechanisms by which PR achieves functional improvements in IPF. We previously observed that values for peak rate of oxygen uptake $\left(\dot{\mathrm{VO}}_{2}\right)$ in people with IPF are half those of similarly aged healthy individuals, whilst their peak heart rate (HR) is $20 \%$ lower and their kinetic HR responses to heavy intensity exercise 2.5 times slower [8] (McNarry et al., 2017). These results imply reduced tissue oxygen utilization following the onset of exercise, which in large part results from impeded central (cardiovascular) oxygen delivery. However, whether PR can improve systemic oxygen delivery and utilization in people with IPF and other causes of UIP is not known.

The single best measure of aerobic fitness (capacity for oxygen utilisation) is considered to be maximal oxygen uptake $\left(\dot{\mathrm{V}}_{2 \max }\right.$ ) but in practice, its measurement can be difficult for example, in older people with impaired mobility or those with cardiorespiratory disease who have limited functional capacity [9] (Jones et al., 2017). Other traditional measures of aerobic exercise fitness such as $\dot{\mathrm{VO}}_{2}$ at the ventilatory anaerobic threshold (VAT) can be similarly problematic in such populations and alternative techniques have been developed to help overcome these difficulties (Hollenberg \& Tagger, 2000 [10], Baba et al., 1999 [11]). For example, the "oxygen uptake efficiency slope" (OUES) quantifies the body's oxygen uptake and extraction efficiency (the rate of increase in oxygen uptake $\left(\dot{\mathrm{VO}}_{2}\right)$ in response to increases in minute ventilation (VE) during incremental exercise. The relationship between $\mathrm{VE}$ and $\dot{\mathrm{VO}}_{2}$ is determined by the pulmonary dead-space/tidal volume ratio $(\mathrm{Vd} / \mathrm{Vt})$, the arterial partial pressure of carbon dioxide $\left(\mathrm{pCO}_{2}\right)$, and the respiratory exchange ratio (RER). Consequently, OUES facilitates a mechanistic assessment of two fundamental cardiovascular processes during exercise. Firstly, pulmonary perfusion (related to $\mathrm{Vd} / \mathrm{Vt}$ ) and secondly, perfusion of working muscles (related to $\mathrm{pCO}_{2}$ and RER) in response to underlying metabolic demand. The OUES has several advantages over other measures of aerobic exercise performance: it can be determined accurately from submaximal exercise protocols (below 60\% - 75\% functional reserve) [12] (R. Baba et al., 1996) [13] (R. Baba, 2000) [10] (M. Hollenberg \& I. B. Tager, 2000) yet is strongly correlated with $\mathrm{VO}_{2}$ max; it is completely objective (tester-independent) and it has better inter-protocol agreement [12] (R. Baba et al., 1996) [13] (R. Baba, 2000). However, whilst OUES has been quantified in various populations including children, adults with heart disease and elite athletes [10] [14] [15] [16] (Davies et al., 2006; M. Hollenberg \& I. B. Tager, 2000; Marinov, Mandadzhieva, \& Kostianev, 2007; Rowland \& Cunningham, 1992), it has not been used to study patients with chronic lung disease. 
An alternative to OUES is the analysis of so-called "pulmonary oxygen uptake kinetics" which involves mathematical modelling of the rate of change of oxygen utilisation in response to a sudden change in exercise workload. This provides insight into an individual's ability to absorb and utilize oxygen to fuel a change in metabolic activity [17] (Whipp \& Ward, 1990). The procedure involves fitting either a mono-exponential or bi-exponential model to the empirical temporal data, with the time-constant of the best-fit model reflecting an individual's oxygen utilisation response rate. Several studies have explored the influence of PR on oxygen uptake kinetics in Chronic Obstructive Pulmonary disease (COPD) [4] [18] (Spruit, 2013) (Puente-Maestu et al., 2000) examined the effects of supervised and unsupervised eight-week PR programmes on a mono-exponential oxygen uptake model and observed an increase in the mean endurance time at a work rate equivalent to $70 \%$ of $\mathrm{VO}_{2}$ Max among two groups and $\mathrm{CO}_{2}$ output, minute ventilation and heart rate were also observed to have increased post training. Similar studies in IPF patients have been limited [5] (Bolton, 2013). McNarry et al., [8] (McNarry et al., 2017) compared the oxygen uptake response in patients with emphysema and IPF and found both displayed $\mathrm{VO}_{2}$ kinetics that were similarly slow in comparison to controls. A study of 11 patients with IPF observed improvement in treadmill exercise time and maintained oxygen consumption $\left(\mathrm{VO}_{2}\right)$ following a three-month $\mathrm{PR}$ program, whereas controls suffered a decline in $\mathrm{VO}_{2}$ kinetics. Other studies have reported improvement in exercise tolerance, dyspnoea and quality of life in people with IPF who undergo PR [1] [19] [20] [21] (Vainshelboim et al., 2015; Vainshelboim et al., 2014) (Society, 2000) (Raghu et al., 2011), but the physiological mechanisms by which such improvements occur are not understood. In this context, the aim of the present study was to quantify dynamic pulmonary function (oxygen uptake kinetics) and cardiac function (heart rate kinetics) in response to metabolic challenge from two different modes of exercise, before and after a bespoke, eight-week PR programme specifically designed to meet the needs of people with interstitial lung disease.

\section{Methods}

\subsection{Participants}

The study was performed in accordance with the Declaration of Helsinki. Ethical approval to conduct the study was obtained from Wales Research Ethics Committee 6, on $03^{\text {rd }}$ November 2016, which is a part of the UK Health Departments' Research Ethics Service. Approval and permission to execute the study was obtained from the local health board (Abertawe Bro Morgannwg University Health Board). Recruitment was led by a consultant chest physician, who informed eligible patients about the study (including its purpose, protocol, expected commitment and the possible risks of involvement). Patients attending the Interstitial Lung Disease Clinic at Moriston Hospital, Swansea, who had been diagnosed with UIP (either IPF or UIP associated with rheumatoid disease) by a Multi-Disciplinary Team, according to the American Thoracic Society (ATS) 
and European Respiratory Society (ERS) guidelines [1] (Raghu et al., 2011) were eligible to participate. All recruited individuals provided their written informed consent to participate in the study. Eighteen individuals (13 males, 5 female) agreed to participate in the study but three males later withdrew from the rehabilitation programme for personal reasons. Patients continued with their usual medication regimes whilst participating in the study. The patients' demographics are shown in Table 1 . The majority (14 of 15) of our patients had IPF whilst 1 had rheumatoid arthritis and a UIP pattern on CT scan.

Exclusion criteria:

- Respiratory infection within the previous two months;

- Known coronary or valvular heart disease;

- Any other serious co-morbid condition (e.g. emphysema, uncontrolled diabetes, renal disease) Significant hypoxia (oxygen saturation below 85\%) when breathing air and undergoing conventional screening for a pulmonary rehabilitation programme;

- Musculoskeletal problems that would make it impossible to exercise;

- Current smokers.

\subsection{Experimental Protocol}

Participants were asked to visit the lung function laboratory at Morriston Hospital, Swansea, for baseline physiological assessment. They were also asked to refrain from consuming alcohol 24 hours before each test, to not consume caffeine six hours prior to the tests, but otherwise to have eaten and consumed fluids as normal. All tests were conducted on the same time of the day \pm 1 hour.

On the first day of testing the participants undertook six-minute walk test to determine their functional (walking) capacity (six-minute walk distance, 6 MWD), in line with the ATS guidelines [22] (Torrey, 2002). Forced expiratory volume in one second (FEV1) and forced vital capacity (FVC) were measured using the spirometry test function of the online respiratory gas analyser. On a separate day they were asked to complete an incremental bicycle exercise test on a stationary bicycle (ergometer) (VIAsprint ${ }^{\mathrm{TM}}$ 150P Ergometer, Vyaire Medical, IL, USA) in accordance with methods described by McNarry et al. [8] (McNarry et al., 2017) and Arena et al. [23] (Arena, 2011). Participants first completed three minutes of cycling with zero resistance, after which the work rate was increased at a rate of $10-15 \mathrm{~W} \cdot \mathrm{min}^{-1}$, depending on the individual's age-predicted maximum workload. They were always asked to maintain a cadence of 55 - 60 rpm and to continue cycling until they reached their limit of exercise tolerance; the test was stopped by the researchers if a patient desaturated to an oxygen level below $85 \%$. Throughout the test patients were asked to breathe through a facemask which continuously sampled the inspired and expired air using a bidirectional pilot tube flow sensor (MCD Medgraphics Ultima CardiO2; MGC Diagnostics, MN, USA). Breath-by-breath data were analysed using the Breeze software package (Version 6.4.1, Medical Graphics, MN, USA). The maximum rate 
of oxygen uptake ( $\dot{\mathrm{V}}_{2 \max }$ ) was determined by averaging the $\dot{\mathrm{V}} \mathrm{O}_{2}$ values from the final 10 seconds before the limit of exercise was reached. Aerobic threshold (AT) was identified by two physiologists using a combination of the two methods used by Castro et al. [24] (Castro, Pedrosa, Chabalgoity, Sousa, \& Nobrega, 2010): Firstly, using the point of upward inflection of the $\dot{\mathrm{V}}_{2}$ vs $\mathrm{VO}_{2}$ curve and secondly, using the onset of a consistent increase in ventilatory equivalent for oxygen $\left(\mathrm{Ve} / \dot{\mathrm{VO}}_{2}\right)$ occurred with no increase in the ventilatory equivalent for carbon dioxide $\left(\mathrm{Ve} / \dot{\mathrm{V}} \mathrm{CO}_{2}\right)$. Blood oxygen saturation was continuously monitored (Nonin Model 7500, Plymouth, MN, USA).

Participants were asked to perform a repeat bicycle exercise test on a separate day. This consisted of a six-minute warmup with no external resistance, followed by an immediate transition to a fixed uniform workload. The uniform workload was calculated as $40 \%$ of the difference between the AT and the $\mathrm{VO}_{2 \mathrm{Max}}(\Delta 40 \%)$ [8] (McNarry et al., 2017), using data obtained from the incremental exercise test. This workload was maintained for six minutes or until the participant reached their limit of tolerance, after which there was an immediate transition back to cycling with no external resistance for a further three minutes. Participants were asked to maintain a pedalling cadence of $55-60 \mathrm{rpm}$ throughout each stage of the test.

A Holter ECG recorder (Pathfinder/Lifecard Digital CF system; Spacelabs Medical Ltd., UK) provided continuous ECG data throughout each exercise test, from which heart rate was derived.

\subsection{Pulmonary Rehabilitation Program}

Following baseline measurements participants began a six-week PR programme (two sessions per week, two hours per session) under the direction of the Physiotherapy Department at Moriston Hospital, Swansea. This programme consisted of ten minutes of "warming up" (20 sets of "marching on the spot", "heal digs", "knee lifts", "shoulder rolls", "knee bends" and "high knees") followed by a range of strength training exercises ("bicep curls", "triceps extension", "dumbbell press", "dumbbell fly", "leg extension" and "dumbbell squats") and cardiovascular exercises (choice of treadmill, bicycle ergometer or cross-trainer exercise). Each patient was asked to choose a weight that they could safely handle at the beginning of the rehabilitation for use in each exercise, and they were encouraged to increase their weights on each day of rehabilitation as the programme progressed. For the cardiovascular exercise patients were asked to select a mode of exercise with which they were comfortable, and this was maintained with increasing workload (resistance) as rehabilitation progressed. Each strength exercises lasted five minutes. The cardiovascular exercise lasted ten minutes, followed by a ten-minute cool-down period which included sets of "hip flexor stretches", "thigh stretch", "calf stretch" and "lower back stretch". Upon completion of the PR programme, patients were asked to return to the hospital for repeat physiological and functional assessments as described above. 


\section{Data Analysis}

\subsection{Oxygen Uptake Kinetics Analysis}

Each participant's $\dot{\mathrm{VO}}_{2}$ response was first plotted to check its structure and to identify any artefacts in the data (which could arise, for example, owing to swallowing or coughing). Individual responses were then normalized to the end-exercise value (by dividing all $\dot{\mathrm{V}}_{2}$ values by the maximum $\dot{\mathrm{V}}_{2}$ value within the last 30 seconds of exercise). In accord with previous studies [8] (McNarry et al., 2017) we modelled the individual temporal $\dot{\mathrm{VO}}_{2}$ responses using a single exponential model (Equation (1)):

$$
\dot{\mathrm{V}} \mathrm{O}_{2}=A \cdot\left(1-\mathrm{e}^{-(t-\delta / \tau)}\right)
$$

where " $t$ " is time, " $A$ " is the amplitude of the response (maximum value of $\dot{\mathrm{V}} \mathrm{O}_{2}$, with baseline $\dot{\mathrm{V}} \mathrm{O}_{2}$ immediately prior to exercise set to zero), " $\delta$ " is a time delay and " $\tau$ " is the time constant. We did not observe evidence of so-called Phase I type behaviour (an exponential rise in $\dot{\mathrm{V}}_{2}$ attributed to an increase in pulmonary blood flow at the onset of exercise [25] [26] (Cummin, Iyawe, Mehta, \& Saunders, 1986) (Bell, Paterson, Kowalchuk, Padilla, \& Cunningham, 2001) in any of our data so we chose to remove the time-delay $(\delta=0)$. Neither did we observe Phase III ( $\dot{\mathrm{VO}}_{2}$ slow component) behaviour, in common with previous work [8] (McNarry et al., 2017). We therefore chose to perform our analysis solely in terms of the mean response time (MRT), which reflects the time course of the entire $\dot{\mathrm{V}} \mathrm{O}_{2}$ response (MRT is thus equivalent to $\tau$ in Equation (1)). The individually normalised $\dot{\mathrm{VO}}_{2}$ responses were modelled using Matlab's Curve Fitting Tool (non-linear least squares method) to determine variables $\mathrm{A}$ and $\tau$ and their associated $95 \%$ confidence bounds, as well as the goodness of fit of the model. To facilitate group averaging of the individual responses all data were interpolated so that $\dot{\mathrm{VO}}_{2}$ were defined uniformly at 0.5 -second intervals. The group-averaged $\dot{\mathrm{V}}_{2}$ response was then separately modelled (using the model and criteria described above) for pre- and post-rehabilitation responses. Finally, group-averaged responses were re-sampled at 30 -second intervals to facilitate statistical comparison at 12 time-points (over six minutes) during exercise.

\subsection{Oxygen Uptake Efficiency Slope (OUES)}

OUES was calculated using the linear relationship between $\dot{\mathrm{VO}}_{2}$ and the logarithm of Ve measured during the incremental exercise tests. This relationship can be expressed as:

$$
\dot{\mathrm{V}} \mathrm{O}_{2}=a \cdot \log _{10} V e+b
$$

where OUES is equal to the slope "a" [10] [27] (Akkerman et al., 2010; R. Baba, 2000) (M. Hollenberg \& I. B. Tager, 2000). Signed Rank Wilcoxon tests were conducted to assess the influence of the pulmonary rehabilitation program in OUES.

\subsection{Statistical Analysis}

Matlab (version 2017b, The Mathworks, Cambridge, UK) was used to perform 
descriptive statistical analysis. The One-Sample Kolomogorov-Smirnov Test was used to test the null hypothesis that data obeyed a Normal distribution. If this assumption was violated, then the influence of the rehabilitation intervention on each variable was assessed using Wilcoxon Signed Rank tests. One-sample Kolmogorov-Smirnov tests showed that none of the quantified variables were Normally-distributed. Consequently, the Wilcoxon Signed-rank test was used to assess the influence of pulmonary rehabilitation for each variable. Statistical significance was assumed at the $\mathrm{p}<0.05$ level.

\section{Results}

Table 1 summarises the respiratory variables measured before and after pulmonary rehabilitation. Table 2 and Table 3 summarise the physiological responses

Table 1. Participants' anthropometric characteristics and resting lung function values.

\begin{tabular}{cc}
\hline & Mean (SD); Range \\
\hline Age (years) & $69.8(7.1) ; 55-83$ \\
Gender & 10 Male, 5 Female \\
BMI $\left(\mathrm{kg} \cdot \mathrm{m}^{-2}\right)$ & $30.3(6.5) ; 20.8-47.7$ \\
$\mathrm{FEV}_{1}(\mathrm{~L})$ & $2.1(0.5) ; 1.1-2.9$ \\
$\mathrm{FEV}_{1}(\%$ predicted $)$ & $78(19) ; 55-130$ \\
FVC $(\mathrm{L})$ & $2.6(0.8) ; 1.5-4.0$ \\
$\mathrm{FVC}(\%$ predicted $)$ & $76(22) ; 52-131$ \\
FEV $/$ FVC & $0.81(0.17) ; 0.62-1.36$ \\
$\mathrm{DL}_{\mathrm{CO}}(\%$ predicted $)$ & $46.7(7.3) ; 35-57$ \\
$\mathrm{~K}_{\mathrm{CO}}(\%$ predicted $)$ & $78.2(11.4) ; 62-95$
\end{tabular}

Table 2. Physiological responses to incremental exercise tests pre- and post-rehabilitation.

\begin{tabular}{|c|c|c|c|}
\hline & Pre: Mean (SD); Range & Post: Mean (SD); Range & $\mathrm{p}$ \\
\hline $\mathrm{Ve}\left(\mathrm{mL} \cdot \mathrm{min}^{-1} \cdot \mathrm{kg}^{-1}\right)$ & $56.0(20.78) ; 22.4-92.3$ & $58.1(18.0), 30.7-90.5$ & 0.53 \\
\hline Peak $\dot{\mathrm{VO}}_{2}\left(\mathrm{~L} \cdot \mathrm{min}^{-1}\right)$ & $1.30(0.28) ; 0.86-1.81$ & $1.28(0.30) ; 0.83-1.91$ & 0.96 \\
\hline Peak $\dot{\mathrm{V}}_{2} \quad\left(\mathrm{~mL} \cdot \mathrm{min}^{-1} \cdot \mathrm{kg}^{-1}\right)$ & $15.7(4.4) ; 10.0-28.4$ & $15.2(4.4) ; 8.4-26.2$ & 0.93 \\
\hline Peak HR (bpm) & $120(24) ; 73-159$ & $90(30) ; 46-136$ & $0.0002^{\star}$ \\
\hline Peak Work Rate (Watts) & $105(35) ; 29-158$ & $78(25) ; 35-128$ & $0.0005^{*}$ \\
\hline Time to Peak Work Rate (minutes) & $11.8(2.2) ; 7.3-16.4$ & $11.4(3.0), 6.1-16.4$ & 0.73 \\
\hline OUES $\left(\mathrm{ml} \cdot \mathrm{min}^{-1} \cdot \mathrm{L}^{-1}\right)$ & $1569(206) ; 1275-1943$ & $1509(250) ; 1121-1929$ & 0.14 \\
\hline
\end{tabular}

Table 3. Physiological responses to constant work load tests pre- and post-rehabilitation.

\begin{tabular}{cccc}
\hline & Pre & Post & p \\
\hline $\mathrm{Ve}\left(\mathrm{mL} \cdot \mathrm{min}^{-1} \cdot \mathrm{kg}^{-1}\right)$ & $54.0(15.8) ; 30.3-87.1$ & $48.1(18.5) ; 21.6-78.8$ & 0.25 \\
Peak $\quad \dot{\mathrm{VO}}{ }_{2} \quad\left(\mathrm{~L} \cdot \mathrm{min}^{-1}\right)$ & $1.27(0.29) ;(0.85-1.81)$ & $1.17(0.35) ;(0.63-1.90)$ & 0.21 \\
Peak $\quad \dot{V O}_{2} \quad\left(\mathrm{~mL} \cdot \mathrm{min}^{-1} \cdot \mathrm{kg}^{-1}\right)$ & $15.0(4.6) ; 9.1-28.1$ & $13.8(4.9) ; 6.4-25.6$ & 0.35 \\
Peak HR $(\mathrm{bpm})$ & $117(22) ;(90-151)$ & $120(26) ; 70-148$ & 0.70 \\
\hline
\end{tabular}


to incremental and constant work rate exercise tests pre- and post-rehabilitation. $V e$, peak $\dot{\mathrm{V}}_{2}$, time to peak work rate and OUES were all similar during the incremental tests pre- and post-rehabilitation, but peak $\mathrm{HR}$ and peak work rate were both significantly reduced following rehabilitation. Ve, peak $\dot{\mathrm{VO}}_{2}$ and peak HR were all similar during the pre- and post-rehabilitation constant work rate tests.

Visual inspection of the group-averaged $\dot{\mathrm{V}}_{2}$ responses during the constant work rate tests (Figure 1(a)) suggested an increase in $\dot{\mathrm{V}}_{2}$ post-rehabilitation
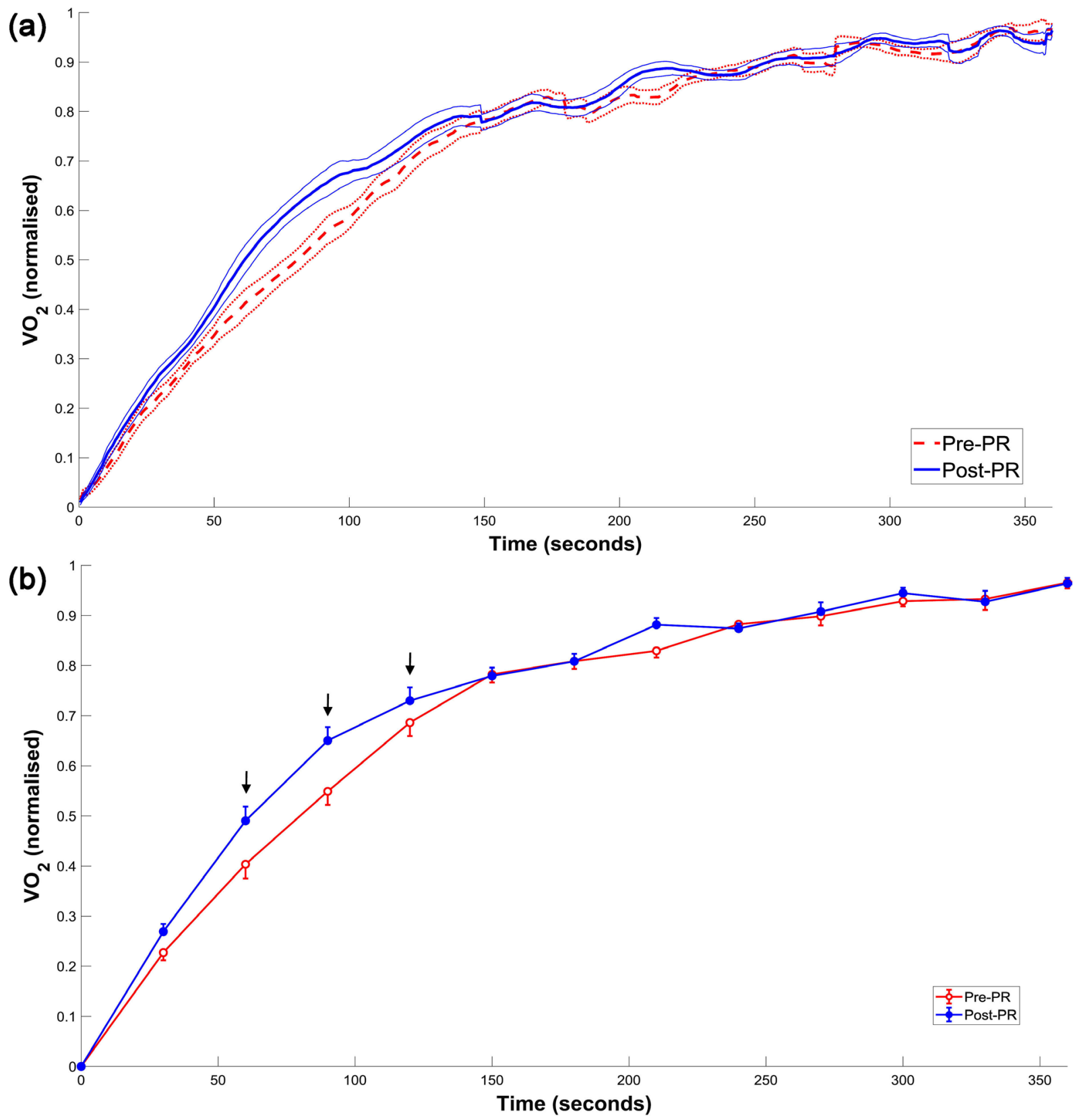

Figure 1. (a) Group-averaged normalised temporal $\dot{\mathrm{VO}}_{2}$ response to constant work rate exercise: re-rehabilitation (red, dashed line) and post-rehabilitation (blue, solid thick line) and corresponding SEM values (pre: red, dotted: post: blue, solid thin); (b) $\dot{\mathrm{VO}}_{2}$ responses from (a) resampled at 30 second intervals; arrows = significant difference pre vs post $(\mathrm{p}<0.05)$. 
during the first two minutes of exercise. Statistical comparison (Wilcoxon signed rank test of $\dot{\mathrm{V}}_{2}$ at each of the 12 time-points in the re-sampled time-series; Figure 1(b)) confirmed that $\dot{\mathrm{V}}_{2}$ was significantly larger post-rehabilitation at times $\mathrm{t}=60,90$ and 120 seconds ( $\mathrm{p}=0.028,0.008$ and 0.034 respectively).

Visual inspection of the group-averaged HR responses during the constant work rate tests (Figure 2(a)) suggested a reduction in HR post-rehabilitation during the first four minutes of exercise. Statistical comparison (Wilcoxon signed
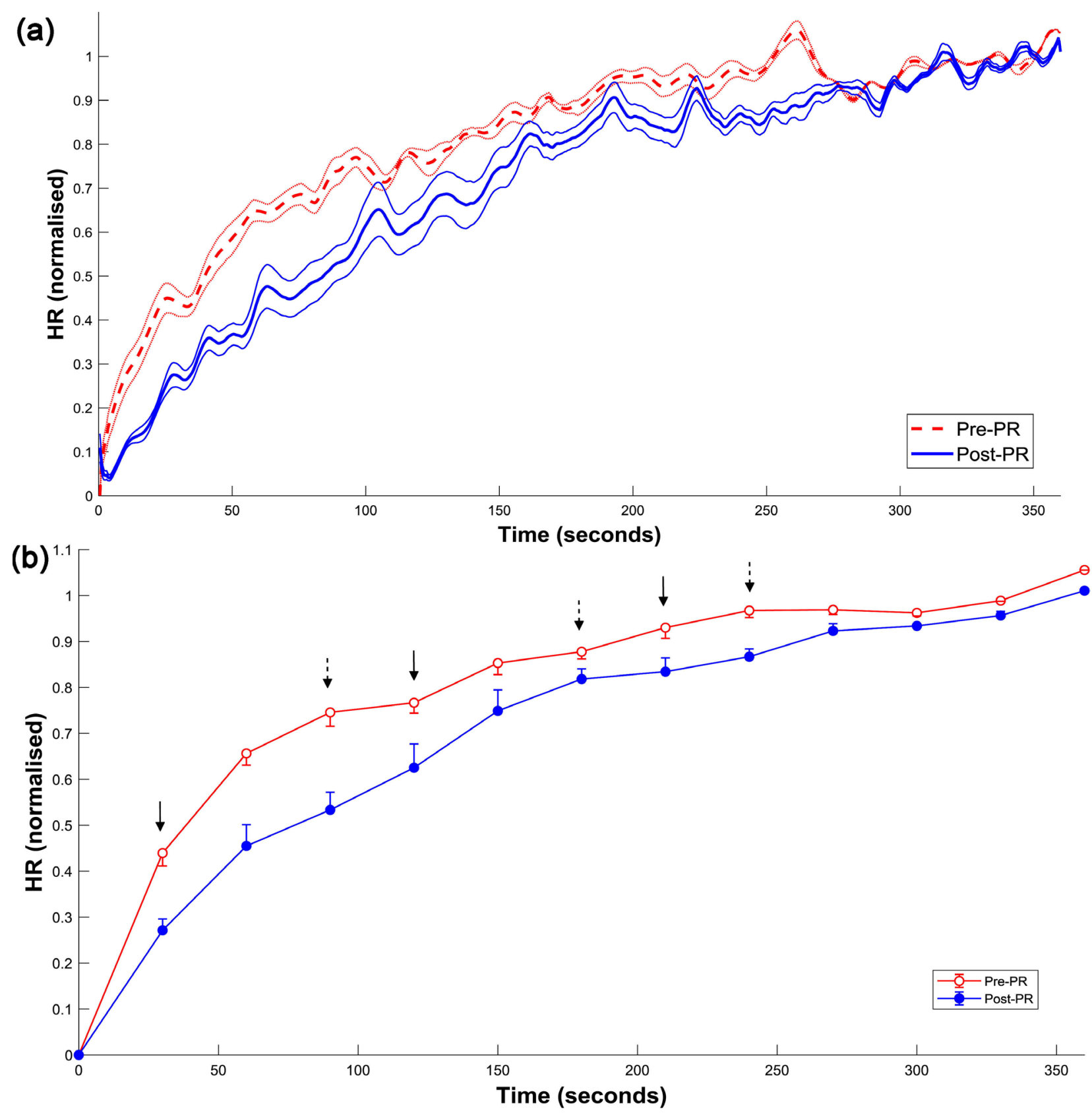

Figure 2. (a) Group-averaged normalised temporal HR response to constant work rate exercise: re-rehabilitation (red, dashed line) and post-rehabilitation (blue, solid thick line) and corresponding SEM values (pre: red, dotted: post: blue, solid thin); (b) HR responses from (a) resampled at 30 second intervals; sold arrows = significant difference pre vs post $(\mathrm{p}<0.05)$; dashed arrows $=$ difference pre vs post $(\mathrm{p}<0.10)$. 
Table 4. Results of group-averaged, normalised $\dot{\mathrm{V}}_{2}$ and $\mathrm{HR}$ modelling.

\begin{tabular}{ccccc}
\hline & \multicolumn{3}{c}{$\dot{\mathrm{VO}}_{2}$} & \multicolumn{2}{c}{$\mathrm{HR}$} \\
\cline { 2 - 5 } & Pre $(95 \% \mathrm{CI})$ & Post $(95 \% \mathrm{CI})$ & Pre $(95 \% \mathrm{CI})$ & Post $(95 \% \mathrm{CI})$ \\
\hline $\mathrm{A}$ & $0.994(0.990,0.998)$ & $0.956(0.954,0.959)$ & $0.97(0.964,0.976)$ & $1.022(1.014,1.029)$ \\
MRT (seconds) & $107.9(106.6,109.1)$ & $85.2(84.4,86.0)$ & $60.5(58.9,62.0)$ & $115.5(113.2,117.8)$ \\
SSE & 0.287 & 0.226 & 1.862 & 0.822 \\
R-squared & 0.994 & 0.995 & 0.940 & 0.983 \\
RMSE & 0.020 & 0.018 & 0.051 & 0.034 \\
\hline
\end{tabular}

SSE = Summed square of residuals (sum of squares due to error); R-squared = squared correlation (coefficient of multiple determination); RMSE = root mean squared error (standard error of the fit).

rank test of $\mathrm{HR}$ at each of the 12 time-points in the re-sampled time-series; Figure 2(b)) confirmed that HR was significantly reduced post-rehabilitation at times $\mathrm{t}$ $=30,120$ and 210 seconds ( $\mathrm{p}$ values $=0.043$ ) and approached a significant difference at times $\mathrm{t}=90,180$ and 240 seconds ( $\mathrm{p}$ values $=0.079$ ).

Table 4 shows the values of the model variables and goodness of fit for the modelled group-averaged, normalised $\dot{\mathrm{VO}}_{2}$ and HR. Goodness of fit measures showed strong adherence of the fitted data to the chosen models, especially for $\dot{\mathrm{V}} \mathrm{O}_{2}$. The improved post-rehabilitation rate of oxygen uptake (noted above) was further reflected by the $21 \%$ reduction in $\tau$ (MRT) in the modelled $\dot{\mathrm{V}}_{2}$ curve. Similarly, the slower post-rehabilitation HR response was reflected by a $48 \%$ increase in $\tau$ (MRT) in the modelled HR curve.

\section{Discussion}

This study was designed to determine whether a bespoke PR programme had any effect on dynamic pulmonary and cardiac responses to metabolic challenge during two modes of exercise, in people with UIP. Following exercise-based PR, rates of oxygen uptake improved significantly during, but not beyond the first two minutes following the onset of heavy-intensity exercise, whilst heart rates were similarly reduced during and beyond this time period. Traditional measures of pulmonary function (minute ventilation and peak rate of respiratory oxygen uptake) were not influenced by PR. We had postulated that the oxygen uptake efficiency slope (OUES) might be a more sensitive measure of aerobic performance plasticity in UIP [12] [27] (Akkerman et al., 2010) (R. Baba et al., 1996) as has been demonstrated in patients with COPD [28] (Ramponi et al., 2018). Interestingly, we found that OUES was unchanged following PR (1569 (pre) vs 1508 (post) $\mathrm{ml} \cdot \mathrm{min}^{-1} \cdot \mathrm{L}^{-1}$ ) in our patients with UIP. Using the prediction Equation (2) in methods [10] (M. Hollenberg \& I. B. Tager, 2000), we estimated OUES for healthy individuals with mean values of age (69.8 years), mass (86.8 $\mathrm{kg})$, height $(1.7 \mathrm{~m})$ and body surface area $\left(1.8 \mathrm{~m}^{2}\right)$ equivalent to our patients; the estimated healthy OUES value was $2010 \mathrm{ml} \cdot \mathrm{min}^{-1} \cdot \mathrm{L}^{-1}$, suggesting an approximate reduction of $25 \%$ in our patients. 
We modelled the temporal oxygen uptake $\left(\dot{\mathrm{V}}_{2}\right)$ response to heavy intensity exercise, characterising the response dynamics in terms of the mean response time (MRT). The MRT for $\dot{\mathrm{V}}_{2}$ in our participants was 108 seconds prior to rehabilitation, similar to the findings of McNarry et al [8] (McNarry et al., 2017) This is substantially slower than that observed for similarly aged healthy individuals [17] [29] (Whipp \& Ward, 1990) (Breese, Barker, Armstrong, Jones, \& Williams, 2012). We also observed a $21 \%$ reduction in MRT to 85 seconds in patients who completed the PR programme. This demonstrates a substantially faster oxygen uptake response to metabolic challenge which was most noteworthy during the initial 2 minutes of exercise.

Although the peak heart rates we observed during heavy-intensity exercise were similar before and after PR, the time to attain this peak value was almost twice as slow following PR (MRT $=60.5$ vs. 115.5 seconds). These times are similar to those in three patients with IPF reported by McNarry et al and substantially greater than those of $12 \mathrm{~s}-60 \mathrm{~s}$ (2SD) reported for healthy individuals [8] (McNarry et al., 2017). Furthermore, we noted sustained post-PR reductions in heart rate within the first four minutes of exercise.

It is interesting to speculate on the mechanisms by which PR might improve oxygen uptake responses in UIP. Given the usually severe and progressive nature of the pathological abnormalities in UIP, it would seem that improvements in pulmonary mechanical function or alveolar-capillary gas-exchange are unlikely. More plausible is the notion that PR may increase systemic arterial-tissue oxygen exchange and/or influence cardiovascular function to improve systemic oxygen delivery and usage. Exercise-induced adaptations of any of these might be contributing factors to the improved oxygen uptake dynamics we observed during exercise. Previous studies of patients with COPD suggest that oxygen delivery to muscles (and hence impaired HR kinetics-a proxy for cardiac output and blood flow) might be the limiting factor in $\dot{\mathrm{V}}_{2}$ kinetics. Similarly, McNarry et al., [8] (McNarry et al., 2017) interpreted slower $\mathrm{HR}$ and $\dot{\mathrm{VO}}_{2}$ kinetics as reflecting a limitation of central oxygen delivery in both COPD and IPF. In the present study, we observed a combination of improved (faster) oxygen uptake and diminished (slower) heart rate in response to exercise in patients after completion of the PR programme. One likely explanation for our findings is that PR improves cardiac efficiency either through a myocardial training effect or by normalisation of intrathoracic pressures by learning more efficient breathing techniques during PR. Notably, OUES (the ratio of oxygen uptake to minute ventilation) was unchanged by rehabilitation. Since OUES is a function of blood perfusion to both working muscles and to the lungs [12] [16] [30] (R. Baba et al., 1996) (Rowland \& Cunningham, 1992) (Weber, Kinasewitz, Janicki, \& Fishman, 1982) it seems unlikely that changes in pulmonary or systemic blood flow (nor central cardiac function) caused the improved oxygen uptake in our study. Rather an improvement of gas exchange, probably at the systemic arterial-capillary interface, best explains enhancement of oxygen utilisation following PR. 
This study extends our knowledge of the physiological responses to a bespoke PR programme in people with UIP. We consider our sample of 15 patients to be representative of people with UIP. Nonetheless, the statistical power of our analyses is limited by this relatively small number of participants. In part, our sample size was restricted by the exclusion of patients with co-morbid conditions that might influence respiratory, cardiac or autonomic nervous system function. We were however mindful of the potential influence of too strict a set of exclusion criteria on both recruitment and the applicability of our results to a wider population or people with UIP. With this in mind, we took the decision to include patients with IPF and those with rheumatoid arthritis who had evidence of definite UIP on CT scan of the thorax all of whom are offered the opportunity to undertake the bespoke PR programme if they so wish. Certainly, there was no obvious difference in the physiological data of those with IPF and those with rheumatoid disease. We also did not exclude individuals with controlled diabetes from the study: five (27\%) of our patient cohort were taking medication to treat Type II diabetes. Care was taken to ensure that the only change between the monitoring days was the intervening intervention with PR.

\section{Conclusion}

As far as we are aware, this is the first study to assess the physiological changes in cardiorespiratory function across maximal exercise ranges, before and after a bespoke programme of $\mathrm{PR}$, in people with UIP. We applied novel approaches to evaluate these changes and provide a comprehensive profile of cardiorespiratory performance in UIP and the improvements PR can deliver. Specifically, PR improved the rate of oxygen uptake during heavy-intensity exercise, despite heart rates being substantially lower during this time implying improvement in cardiac pumping efficiency. This suggests that PR may increase systemic arterial-tissue oxygen exchange and/or influence cardiovascular function to improve systemic oxygen delivery. Our findings suggest that individuals with UIP should find it easier to perform the activities of daily life including those requiring substantial metabolic demands, after undertaking an appropriately designed PR programme.

\section{Acknowledgements}

We would like to express our thanks for the support of Caroline Evered, Adrian Paton, Helen Davies, Marie Clare Oliver (Pulmonary Rehabilitation Team, ABMU Health Board), who conducted the rehabilitation programme; Dr. Melitta McNarry (Swansea University) for contributing to study design.

\section{Conflicts of Interest}

The authors declare no conflicts of interest regarding the publication of this paper.

\section{References}

[1] Raghu, G., Collard, H.R., Egan, J.J., Martinez, F.J., Behr, J., Brown, K.K. and Schünemann, H.J. (2011) An Official ATS/ERS/JRS/ALAT Statement: Idiopathic 
Pulmonary Fibrosis: Evidence-Based Guidelines for Diagnosis and Management. American Journal of Respiratory and Critical Care Medicine, 183, 788. https://doi.org/10.1164/rccm.2009-040GL

[2] Ley, B., Collard, H.R. and King Jr., T.E. (2011) Clinical Course and Prediction of Survival in Idiopathic Pulmonary Fibrosis. American Journal of Respiratory and Critical Care Medicine, 183, 431-440. https://doi.org/10.1164/rccm.201006-0894CI

[3] Gross, T.J. and Hunninghake, G.W. (2001) Idiopathic Pulmonary Fibrosis. The New England Journal of Medicine, 345, 517-525. https://doi.org/10.1056/NEJMra003200

[4] Spruit, M.A., et al. (2013) An official American Thoracic Society/ European Respiratory Society Statement: Key Concepts and Advances in Pulmonary Rehabilitation. American Journal of Respiratory and Critical Care Medicine, 188, e13. https://doi.org/10.1164/rccm.201309-1634ST

[5] Bolton, C.E., et al. (2013) British Thoracic Society Guideline on Pulmonary Rehabilitation in Adults: Accredited by NICE. Thorax, 68, ii1-ii30.

https://doi.org/10.1136/thoraxjnl-2013-203808

[6] Dowman, L., Hill, C.J. and Holland, A.E. (2014) Pulmonary Rehabilitation for Interstitial Lung Disease. The Cochrane Database of Systematic Reviews, 10, CD006322. https://doi.org/10.1002/14651858.CD006322.pub3

[7] Tonelli, R., Cocconcelli, E., Lanini, B., Romagnoli, I., Florini, F., Castaniere, I. and Clini, E.M. (2017) Effectiveness of Pulmonary Rehabilitation in Patients with Interstitial Lung Disease of Different Etiology: A Multicenter Prospective Study. BMC Pulmonary Medicine, 17, Article number: 130. https://doi.org/10.1186/s12890-017-0476-5

[8] McNarry, M.A., Harrison, N.K., Withers, T., Chinnappa, N. and Lewis, M.J. (2017) Pulmonary oxygen uptake and muscle deoxygenation kinetics during Heavy Intensity Cycling Exercise in Patients with Emphysema and Idiopathic Pulmonary Fibrosis. BMC Pulmonary Medicine, 17, Article number: 26.

https://doi.org/10.1186/s12890-017-0364-Z

[9] Jones, S., Tillin, T., Williams, S., Coady, E., Chaturvedi, N. and Hughes, A.D. (2017) Assessment of Exercise Capacity and Oxygen Consumption Using a 6 Min Stepper Test in Older Adults. Frontiers in Physiology, 8, 408. https://doi.org/10.3389/fphys.2017.00408

[10] Hollenberg, M. and Tager, I.B. (2000) Oxygen Uptake Efficiency Slope: An Index of Exercise Performance and Cardiopulmonary Reserve Requiring Only Submaximal Exercise. Journal of the American College of Cardiology, 36, 194-201. https://doi.org/10.1016/S0735-1097(00)00691-4

[11] Baba, R., Tsuyuki, K., Kimura, Y., Ninomiya, K., Aihara, M., Ebine, K. and Nagashima, M. (1999) Oxygen Uptake Efficiency Slope as a Useful Measure of cardiorespiratory Functional Reserve in Adult Cardiac Patients. European Journal of Applied Physiology and Occupational Physiology, 80, 397-401. https://doi.org/10.1007/s004210050610

[12] Baba, R., Nagashima, M., Goto, M., Nagano, Y., Yokota, M., Tauchi, N. and Nishibata, K. (1996) Oxygen Uptake Efficiency Slope: A New Index of Cardiorespiratory Functional Reserve Derived from the Relation between Oxygen Uptake and Minute Ventilation during Incremental Exercise. Journal of the American College of Cardiology, 28, 1567-1572. https://doi.org/10.1016/S0735-1097(96)00412-3

[13] Baba, R. (2000) The Oxygen Uptake Efficiency Slope and Its Value in the Assessment of Cardiorespiratory Functional Reserve. Congestive Heart Failure, 6, 256-258. https://doi.org/10.1111/j.1527-5299.2000.80164.x 
[14] Davies, L.C., Wensel, R., Georgiadou, P., Cicoira, M., Coats, A.J., Piepoli, M.F. and Francis, D.P. (2006) Enhanced Prognostic Value from Cardiopulmonary Exercise Testing in Chronic Heart Failure by Non-Linear Analysis: Oxygen Uptake Efficiency Slope. European Heart Journal, 27, 684-690. https://doi.org/10.1093/eurheartj/ehi672

[15] Marinov, B., Mandadzhieva, S. and Kostianev, S. (2007) Oxygen-Uptake Efficiency Slope in Healthy 7- to 18-Year-Old Children. Pediatric Exercise Science, 19, 159-170. https://doi.org/10.1123/pes.19.2.159

[16] Rowland, T.W. and Cunningham, L.N. (1992) Oxygen Uptake Plateau during Maximal Treadmill Exercise in Children. Chest, 101, 485-489. https://doi.org/10.1378/chest.101.2.485

[17] Whipp, B.J. and Ward, S.A. (1990) Physiological Determinants of Pulmonary Gas Exchange Kinetics during Exercise. Medicine \& Science in Sports \& Exercise, 22, 62-71. https://doi.org/10.1249/00005768-199002000-00011

[18] Puente-Maestu, L., Sanz, M., Sanz, P., Ona, J.R.D., Rodriguez-Hermosa, J. and Whipp, B. (2000) Effects of Two Types of Training on Pulmonary and Cardiac Responses to Moderate Exercise in Patients with COPD.

[19] Vainshelboim, B., Oliveira, J., Fox, B.D., Soreck, Y., Fruchter, O. and Kramer, M.R. (2015) Long-Term Effects of a 12-Week Exercise Training Program on Clinical Outcomes in Idiopathic Pulmonary Fibrosis. Lung, 193, 345-354. https://doi.org/10.1007/s00408-015-9703-0

[20] Vainshelboim, B., Oliveira, J., Yehoshua, L., Weiss, I., Fox, B.D., Fruchter, O. and Kramer, M.R. (2014) Exercise Training-Based Pulmonary Rehabilitation Program Is Clinically Beneficial for Idiopathic Pulmonary Fibrosis. Respiration, 88, 378-388. https://doi.org/10.1159/000367899

[21] Society, A.T. (2000) Idiopathic Pulmonary Fibrosis: Diagnosis and Treatment. International Consensus Statement. American Thoracic Society (ATS), and the European Respiratory Society (ERS). American Journal of Respiratory and Critical Care Medicine, 161, 646-664. https://doi.org/10.1164/ajrccm.161.2.ats3-00

[22] Torrey B. (2002) ATS Guidelines for the Six-Minute Walk Test (American Thoracic Society Committee on Proficiency Standards for Clinical Pulmonary Function Laboratories). American Family Physician, 66, 904.

[23] Arena, R. and Sietsema, K.E. (2011) Cardiopulmonary Exercise Testing in the Clinical Evaluation of Patients with Heart and Lung Disease. Circulation, 123, 668-680. https://doi.org/10.1161/CIRCULATIONAHA.109.914788

[24] Castro, R.R., Pedrosa, S., Chabalgoity, F., Sousa, E.B. and Nobrega, A.C. (2010) The Influence of a Fast Ramp Rate on Peak Cardiopulmonary Parameters during Arm Crank Ergometry. Clinical Physiology and Functional Imaging, 30, 420-425. https://doi.org/10.1111/j.1475-097X.2010.00958.x

[25] Cummin, A.R., Iyawe, V.I., Mehta, N. and Saunders, K.B. (1986) Ventilation and Cardiac Output during the Onset of Exercise, and during Voluntary Hyperventilation, in Humans. The Journal of Physiology, 370, 567-583.

https://doi.org/10.1113/jphysiol.1986.sp015951

[26] Bell, C., Paterson, D.H., Kowalchuk, J.M., Padilla, J. and Cunningham, D.A. (2001) A Comparison of Modelling Techniques Used to Characterise Oxygen Uptake Kinetics during the On-Transient of Exercise. Experimental Physiology, 86, 667-676. https://doi.org/10.1113/eph8602150

[27] Akkerman, M., van Brussel, M., Hulzebos, E., Vanhees, L., Helders, P.J. and Takken, T. (2010) The Oxygen Uptake Efficiency Slope: What Do We Know? Journal of 
Cardiopulmonary Rehabilitation and Prevention, 30, 357-373.

https://doi.org/10.1097/HCR.0b013e3181ebf316

[28] Ramponi, S., Tzani, P., Aiello, M., Marangio, E., Clini, E. and Chetta, A. (2018) Pulmonary Rehabilitation Improves Cardiovascular Response to Exercise in COPD. Respiration, 86, 17-24. https://doi.org/10.1159/000348726

[29] Breese, B.C., Barker, A.R., Armstrong, N., Jones, A.M. and Williams, C.A. (2012) The Effect of Baseline Metabolic Rate on Pulmonary $\mathrm{O}_{2}$ Uptake Kinetics during Very Heavy Intensity Exercise in Boys and Men. Respiratory Physiology \& Neurobiology, 180, 223-229. https://doi.org/10.1016/j.resp.2011.11.013

[30] Weber, K.T., Kinasewitz, G.T., Janicki, J.S. and Fishman, A.P. (1982) Oxygen Utilization and Ventilation during Exercise in Patients with Chronic Cardiac Failure. Circulation, 65, 1213-1223. https://doi.org/10.1161/01.CIR.65.6.1213 